334 Ka ưf fmann: Beitrag zu den Substitutionsgesetzen etc.

pharmakologischen Inaugural-Dissertation an der Kaiserl. Universität Dorpat über den „Liber fundamentorum pharmacologiae des Abu Mansur Muwaffak ben Ali Harawi“ nebensächlich den Persern des 10. Jahrhunderts nach Chr. den Gebrauch von Zinkwannen zuzuschreiben versucht.

Die von Herrn Dr. F. C. Andreas und mir angestellte eingehende Untersuchung läßt indes nichts für Zink, wohl aber alles für Kupfer in diesem Falle sprecben. Die „Mitteilungen zur Geschichte der Med. u. d. Naturwissenschaften“" (Hamburg-Leipzig, Voss) werden auch hierüber Näheres bringen.

Berlin, im Februar 1903.

\title{
Ein Beitrag zu den Substitutionsgesetzen bei aromatischen Verbindungen;
}

\author{
von \\ Hugo Kauffmann.
}

(Mitteilung aus dem Laboratorium für allgemeine Chemie der Technischen Hochschule zu Stuttgart.)

Im Septemberheft dieses Journals erschien ein sehr interessanter Versuch Flürscheims, die Substitutionsvorgänge im Benzol- und Naphtalinkern zu erklären. ${ }^{1}$ ) Diese Arbeit ist für mich von besonderem Wert, da ich mich schon seit längerer Zeit mit einem ähnlichen Thema befasse. Darum erscheint es mir erforderlich, denselben Gegenstand auch von meinem Gesichtspunkt aus zu beleuchten. Die darzulegende Auffassung steht in keinem Widerspruche zu der Flürscheims und könnte vielleicht eher als eine Ergänzung derselben angesehen werden

1. Sobald von Substitutionsvorgängen die Rede ist, ist zu beachten, daß es sich nicht nur um die Stellung oder den

1) Dies. Journ. [2] 66, 321 . 
Ka uff fmann: Beitrag zu den Substitutionsgesetzen etc. 335

chemischen Ort, in welchen ein neuer Substituent eintritt, handelt, sondern gleichzeitig um die ebenso wichtige Frage, geht die Substitution leicht oder schwer vor sich. Es genügt z. B. nicht zu wissen, daß die Aniline vorzugsweise in orthound para-, die Nitrobenzole vorzugsweise in meta-Stellung substituiert werden; man muß unbedingt noch berücksichtigen, da $B$ die ersteren sebr leicht, die letzteren dagegen sehr bedeutend viel schwieriger einer Substitution unterliegen. Man muB unabweislich noch die Tatsache in Rechnung ziehen, daB die Aminogruppen das Ringsystem des Benzols lockern und allen möglichen chemischen Einflüssen sehr zugänglich machen, daB hingegen die Nitrogruppen gerade das Gegenteil bewirken, daß sie gerade den Benzolring allen Einflüssen verschließen. Um dies letztere zu zeigen, ist nur nötig, an die Schwierigkeit der Herstellung mehrfach nitrierter Benzole und an die auBerordentlich geringe Neigung nitrierter Kohlenwasserstoffe zur Kondensation mit Aldehyden zu erinnern.

In jedem Benzolderivate wirken also die am Kerne hängenden Substituenten bestimmend für das chemische Verbalten des Ringes. Unabhängig von jeglicher Spekulation über die Konstitution des Benzols erkennt man, daß das Ringsystem des Benzols je nach der Natur dieser Substituenten in verschiedenen Zuständen auftreten kann. Die Verschiedenheit der Zustände bedingt die Verschiedenheit des chemischen Verhaltens. Die Anzahl der möglichen Zustände muß ebenso groß sein, wie die der Benzolderivate; denn a priori ist nicht einzusehen, daß mehrere Benzolabkömmlinge ein und denselben Zustand haben werden.

2. Welche Mittel besitzen wir zur genaueren Charakterisierung des Zustandes eines Benzolderivates?

Zur Zeit sind nur zwei Methoden, beide physikalischchemischer Natur, bekannt und ausgearbeitet. Die erste Methode habe ich vor vier Jahren unabhängig von irgend welcher theoretischen Erwägung ganz durch Zufall aufgefunden. ${ }^{1}$ ) Sie ist anwendbar auf alle verdampfbare oder nur unter ganz geringer Zersetzung destillierende Stoffe. Sie beruht darauf, daß die Intensität des violetten Leuchtens, welches die Dämpfe

1) Z. phys. Chem. 26, 719; 27, 519; 28, 688; Ber. 33, 1725 (1900). 
336 Kauffmann: Beitray zu den Substitutionsgesetzen etc.

vieler aromatischer Stoffe unter dem Einfluß von Teslaströmen zeigen, ein Maßstab der Zustände ist.

Die zweite Methode, die sich auf Untersuchungen W. H. Perkins gründet, ist viel allgemeiner anwendbar, da sie sich auf Flüssigkeiten, event. auch auf gelöste Stoffe bezieht. $^{1}$ ) Überdies hat sie den Vorteil, auch dann noch Aufschluß zu geben, wenn man es mit leuchtunfähigen Stoffen zu tun hat. Nach dieser zweiten Methode hat man die magnetische Molekularrotation eines Stoffes zu ermitteln und zu sehen, um wie viel die beobachtete Rotation größer ist als die berechnete. Diese Differenz, die magneto-optische Anomalie genannt wird, ist gleichfalls ein Maßstab der Zustände.

Die nach diesen beiden, von einander abweichenden $\mathrm{Me}$ thoden erhaltenen Resultate decken sich im wesentlichen.

Mit der Ausarbeitung einer dritten Methode, bei welcher zwischen Zustand und Farbe bestehende Beziehungen benützt werden, bin ich augenblicklich beschäftigt.

Nachstehend gebe ich eine Tabelle der magneto-optischen Anomalie der bekannteren Benzolderivate. Die Zahlen sind so berechnet, daB die magneto-optische Anomalie des Benzols gleich Null gesetzt wurde.

\section{Tabelle I.}

\begin{tabular}{|c|c|c|c|c|c|c|c|c|c|}
\hline$\alpha-\mathrm{p}$ & $\mathrm{rl}$ & amin & 10,967 & Brenzkatechin & & & & & I, \\
\hline Siäthylanilin & & 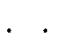 & 8,816 & Pyrogallol & & & & & 1,163 \\
\hline imetbylanili & & . & 8,587 & Phenol & & & & & 0,662 \\
\hline ime & luidin & & 7,518 & lbenzol & & & & & 0,684 \\
\hline $\mathrm{Pl}$ & $\min$ & . & 6,165 & $\mathrm{Be}$ & & & & & $\pm 0,000$ \\
\hline$n-P l$ & $\operatorname{amin}$ & . & 5,617 & enzol & & & & & 866 \\
\hline $0-A n$ & & & 5,250 & dehyd & & & & & $-0,196$ \\
\hline $\mathrm{p}-\mathrm{An}$ & & . & 4,834 & $\mathrm{Be}$ & & & & & 201 \\
\hline Tol & & • & 3,922 & Äth & & & & & - \\
\hline Ani & . & . & 3,821 & enon & & & & & $-0,560$ \\
\hline$-\mathrm{To}$ & 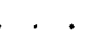 & & 3,069 & chlorid & & & & & $-1,116$ \\
\hline nond & dimethy & I & 2,999 & otoluol & & & & & $-1,730$ \\
\hline$n-T$ & . & - & 2,932 & enzol & & & & & $-2,152$ \\
\hline Ace & . & . & 1,949 & p-Nitrotoluol & & & & & $-2,321$ \\
\hline Jiphenyl & & • & 1,622 & & & & & & \\
\hline
\end{tabular}

Leuchtfähig unter dem EinfluB von Teslaströmen sind alle Stoffe, deren Werte größer als 1 sind, also bis zum Pyrogallol.

1) Ber. 34, 682 (1901). 
Kauffmann: Beitrag zu den Substitutionsgesetzen etc. 337

Die Intensität des Leuchtens läuft ungefähr parallel mit den angeschriebenen Zahlen.

3. Die Tabelle ist sehr lehrreich, denn sie ist ein ziemlich getreuer Spiegel des chemischen Verhaltens. An der Spitze marschieren solche Stoffe, die außerordentlich reaktionsfähig sind, so sehr, daß sie sich schon an der Luft oxydieren. Die Neigung zur Substitution und die zur Kondensation mit Aldehyden ist sehr groß. Substitution findet nur in $\mathrm{p}$ - und o-Stellung statt.

In dem Maße, wie man in der Tabelle herabsteigt, stößt man auf Stofle von geringerer Reaktionsfähigkeit, d. h. auf Stoffe mit widerstandsfähigeren Ringen. Die Substitutierbarkeit in p- und o-Stellung bleibt erhalten bis zum Brombenzol, also bis zu dem Werte - 0,086. Alle nachfolgenden Stoffe, die zum Teil sehr große negative Werte aufweisen, werden in mStellung substituiert. Aus dem Verhalten anderer nicht in die Tabelle aufgenommenen Stoffe kann man schließen, daB die Grenze noch etwas tiefer liegt, wahrscheinlich bei $-0,150$.

Aus der Tabelle geht also aufs deutlichste hervor, daB die Fähigkeit des Substituiertwerdens in para-ortho oder in meta eine Funktion ist des Zustandes des Ringsystems.

4. Durch Salzbildung tritt bei Aminen eine gewaltige Verschiebung ein. In nachstehender Tabelle sind für einige Amine und Chlorhydrate die magneto-optischen Anomalien zusammengestellt. Bei der Berechnung habe ich mich ähnlicher Zahlen bedient, wie sie Perkin bei der Salzbildung aliphatischer Amine beobachtete. ${ }^{1}$ ) $\mathrm{Um}$ aber sicher zu gehen, habe ich als Äquivalent für den bei der Salzbildung durch Salzsäure eintretenden Zuwachs der Rotation nicht den gröBten Wert gewählt, sondern nur die Zahl 4. Infolge dessen dürften die für die Chlorhydrate berechneten Werte der magneto-optischen Anomalie noch etwas zu groß sein, d. h. die Zustandsverschiebung ist noch größer, als durch die Tabelle zum Ausdruck kommt.

1) Journ. of the chem. Soc. 55, 680 (1889).

Journal 1, prakt. Chomie [2] Bd. 67. 
338 Ka uffmann: Beitrag zu den Substitutionsgesetzen etc.

Tabelle II.

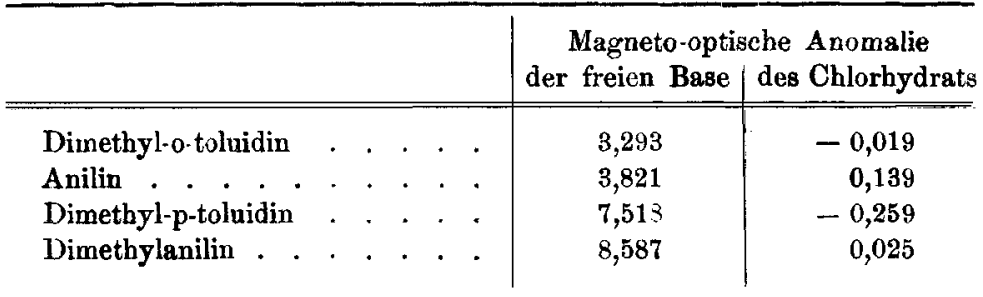

Diese gewaltige Verschiebung, welche die Amine bei der Salzbildung erleiden, erklärt das von der freien Base abweichende Verhalten der Salze. Der Zustand des Ringsystems ist durch die Verbindung mit der Säure so sehr geändert, daB er nunmehr ungefähr dem des Benzaldehyds oder dem von in Tabelle I noch tiefer stehenden Stoffen entspricht. Dadurch wird die Substitution des Anilinsulfates in m-Stellung begreiflich, und verständlich wird auch die sehr viel größere Beständigkeit der Salze von noch so sehr zersetzlichen Aminen.

Nach allem diesem wird man also sicher behaupten dürfen, daB die Wirkung der Aminogruppe auf das Ringsystem des Benzols durch die Salzbildung nicht nur aufgewogen, sondern auch überwogen werden kann.

5. Noch ist darauf aufmerksam zu machen, dab bei Phenolen durch Salzbildung mit Alkalien der Charakter der Verbindung ebenfalls einer sehr starken Veränderung unterliegt. Während eine große Anzahl von Stoffen, wie etwa Pyrogallol, Hydrochinon u. s. w. als freie Phenole ziemlich beständig sind, kann das Gleiche für die Salze nicht mehr behauptet werden. Die Salze oxydieren sich an der Luft ziemlich schnell. Dies rührt davon her, daß bei der Salabildung, ähnlich wie bei Aminen, nur im umgekehrten Sinne, eine außerordentliche Verschiebung des Zustandes erfolgt. In der Tabelle I rücken bei der Salzbildung die Phenole ein großes Stück nach aufwärts, also zu den reaktionsfähigsten Stoffen hin. 